\title{
Variación geográfica de la germinación en Enterolobium cyclocarpum en la costa de Oaxaca, México
}

\author{
Geographic variation in germination of the Enterolobium cyclocarpum \\ of the coast of Oaxaca, Mexico
}

\author{
Héctor Viveros-Viveros ${ }^{\text {a*}}$, Karen Quino-Pascual ${ }^{\mathrm{b}}$, Mario Valerio Velasco-García ${ }^{\mathrm{c}}$, \\ Gabriela Sánchez-Viveros ${ }^{\mathrm{b}}$, Efraín Velasco Bautista ${ }^{\mathrm{d}}$ \\ *Autor de correspondencia: a Universidad Veracruzana, Instituto de Investigaciones Forestales, Parque Ecológico "El Haya", \\ Colonia Benito Juárez, Xalapa, Veracruz, México, C.P. 91070, tel.: 2288421700 Ext. 13964, heviveros@hotmail.com \\ ${ }^{\mathrm{b}}$ Universidad Veracruzana, Facultad de Ciencias Agrícolas, Campus Xalapa, Circuito Gonzalo Aguirre Beltrán, \\ Isleta, 91090 Xalapa, Veracruz, México. \\ ${ }^{\mathrm{c}}$ Colegio de Postgraduados, Campus Montecillo, Postgrado en Ciencias Forestales, \\ Carretera México-Texcoco, Montecillo, Texcoco, Estado de México, México. \\ ${ }^{\mathrm{d}}$ Instituto de Investigaciones Forestales, Agrícolas y Pecuarias (INIFAP), México.
}

\begin{abstract}
SUMMARY
Enterolobium cyclocarpum is recommended in reforestation and ecological restoration programs. However, before starting large-scale plantations some germination parameter form different seed sources should be identified, which can be used for plant production in each location or use elsewhere. In this paper, the variation of some germination parameters from ten provenances of $E$. cyclocarpum from the Coast of Oaxaca, Mexico was explored. Four germination parameters: germination capacity, mean daily germination, peak value and germination value were assessed in the laboratory. From information about geographical location and altitude above sea level of each provenance, climatic variables were obtained and afterward correlated with the germination parameters. Significant differences among provenances were detected for all of the germination parameters. There was not significant relationship among germination parameters and geographic variables, but there was a positive relationship between germination capacity and the proportion of precipitation falling during summer; and between mean daily germination and the total precipitation that falls during the growing season. The germinative capacity varied from 28.2 to $90 \%$; it depended on provenance. The low germination of some provenances can cause problems in their permanence and conservation.
\end{abstract}

Key words: climatic variables, germination parameters, geographic variables, provenances, germinative capacity.

\section{RESUMEN}

Enterolobium cyclocarpum es recomendada en programas de reforestación y restauración ecológica. Sin embargo, antes de realizar plantaciones a gran escala se deben identificar algunos parámetros germinativos de las diferentes procedencias que se utilicen para la producción de planta, sea local o para otros sitios. En el presente trabajo se estudió la variación entre 10 procedencias de parámetros germinativos de semillas de E. cyclocarpum procedentes de la Costa de Oaxaca, México. Se evaluaron cuatro parámetros germinativos en el laboratorio: capacidad germinativa, germinación media diaria, valor pico y valor germinativo. A partir de la información de ubicación geográfica y altitud sobre el nivel del mar de cada procedencia, se obtuvieron variables climáticas de las procedencias, para asociarse a los parámetros germinativos. En todas las variables se detectaron diferencias significativas entre procedencias. La asociación entre los parámetros germinativos y las variables geográficas no fue significativa, pero se encontró asociación positiva entre la capacidad germinativa con la proporción de precipitación que cae durante el verano y entre la germinación media diaria y la precipitación total que ocurre en la estación de crecimiento. La capacidad germinativa varió del 28,2 al $90 \%$, dependiendo de la procedencia. La baja germinación que presentaron algunas procedencias puede ocasionarles problemas en cuanto a su permanencia y conservación.

Palabras clave: capacidad germinativa, parámetros germinativos, variables climáticas, variables geográficas, procedencias.

\section{INTRODUCCIÓN}

Enterolobium cyclocarpum (Jacq.) Griseb. se distribuye en forma natural en México (desde los $25^{\circ} \mathrm{N}$ ), Centroa- mérica y norte de Sudamérica (hasta los $7^{\circ} \mathrm{S}$ ); también se encuentra en Jamaica, Cuba, Trinidad y Guyana (Espejel y Martínez 1979, CATIE 1997). En México, E. cyclocarpum se encuentra ampliamente distribuida en la vertiente del 
golfo desde Tamaulipas hasta Yucatán, y en la Vertiente del Pacífico desde Sinaloa hasta Chiapas (Manzanilla et al. 2001). Su distribución altitudinal va de 0 a $1.200 \mathrm{~m} \mathrm{snm}$ (CATIE 1997).

Esta especie tiene gran importancia económica y social por ser un árbol de usos múltiples en sistemas agroforestales, proporciona productos utilizables en forma de bienes y servicios en las regiones rurales. Por ejemplo, su madera se considera como preciosa y es muy cotizada en la Costa Sur del Pacífico en Guatemala, además de que sirve de albergue para la fauna silvestre (Buch-Texaj 1999). En los estados mexicanos de Chiapas, Guerrero, Michoacán y Morelos, los frutos y semillas de este árbol tradicionalmente se destinan para el consumo humano y como forraje (Serratos-Arévalo 2001). Específicamente en la región Costa de Oaxaca, México, la madera por su resistencia y belleza se usa para muebles, leña y artesanías, la corteza tiene usos medicinales, el fruto se usa como forraje y las semillas tienen usos medicinales (Cano-Hernández y Romero-Guillot 2012).

Es ampliamente recomendada en programas de reforestación, enriquecimiento y restauración ecológica (Vázquez-Yanez et al. 1999). Se espera que las plantaciones forestales de esta especie se incrementen en los próximos años (Benítez-Badillo et al. 2004). Sin embargo, antes de iniciar las plantaciones a gran escala se deben de identificar las procedencias con mayor potencial para producir semilla viable que se pueda utilizar para la producción masiva de planta, asimismo conocer la variación adaptativa de estas procedencias. Esto se puede lograr a través del establecimiento de ensayos de procedencias o poblaciones en los que se evalúe la germinación, la adaptación y el crecimiento en vivero y campo; considerando la procedencia como la fuente geográfica original de la que se obtuvieron las semillas (Zobel y Talbert 1992).

La respuesta germinativa de las semillas varía debido a factores ambientales y geográficos, como por ejemplo la latitud, altitud sobre el nivel del mar, humedad del suelo, temperatura, tipo y densidad de la cobertura arbórea, del grado de disturbio del hábitat dónde las semillas maduran y de la disponibilidad de polen viable previo a la formación del embrión (Ginwal et al. 2005).

En varias especies de plantas se ha encontrado variación entre procedencias en los parámetros germinativos de semillas, por ejemplo, en la capacidad germinativa, valor pico y valor germinativo según Czabator (1962) de Alnus sinuata Rydb. y Betula papyrifera Marsh. (Benowicz et al. 2000, 2001), en la capacidad germinativa (Juárez-Agis et al. 2006) y valor germinativo (Mápula-Larreta et al. 2008) de Pseudotsuga menziesii (Mirb.) Franco y en la capacidad germinativa de E. cyclocarpum (Bertsch 1985) y Abies amabilis [Dougl.] Forbes (Davidson et al. 1996). Sin embargo, se desconoce la relación entre parámetros germinativos con factores geográficos y ambientales de procedencias mexicanas de E. cyclocarpum. Además, algunas poblaciones pudieran presentar problemas germina- tivos, por tanto los trabajos de reforestación necesitarían incluir más esfuerzo en la recolecta de mayores cantidades de germoplasma.

Por lo anterior, los objetivos de la presente investigación son: a) cuantificar la variación entre procedencias de parámetros germinativos de E. cyclocarpum de la costa de Oaxaca, México y b) explorar la asociación de los parámetros germinativos con las variables geográficas y climáticas de las procedencias de E. cyclocarpum de la costa de Oaxaca, México.

\section{MÉTODOS}

Recolección de semillas. Las semillas utilizadas fueron recolectadas de marzo a mayo de 2008, en 10 procedencias de la región Costa de Oaxaca, México (cuadro 1, figura 1). En cada procedencia se seleccionaron de siete a 12 árboles con características fenotípicas deseables, es decir, árboles con fustes rectos, fuste limpio $>2 \mathrm{~m}$ de altura, y libre de plagas y enfermedades (Flores-Flores et al. 2014). Los frutos se recolectaron mediante escalado en los árboles y en algunos casos se recogieron frutos tirados en el suelo, pero se cuidó que correspondieran al árbol seleccionado, procurando que los frutos no estuvieran alejados a más de $1 \mathrm{~m}$ de la copa del árbol seleccionado. Los frutos de cada árbol se almacenaron en costales y se etiquetaron con el nombre de la procedencia y fecha de recolección, para su traslado a las instalaciones de la Universidad del Mar, Campus Puerto Escondido, Oaxaca, México.

Beneficio y almacenamiento de la semilla. Los frutos se secaron al sol directo y se colocaron al fuego aproximadamente a $90{ }^{\circ} \mathrm{C}$ para extraer las semillas; posteriormente se eliminaron las impurezas (semillas dañadas y vanas, trozos de material vegetal y piedras). Por último, las semillas se almacenaron a temperatura ambiente $\left(25^{\circ} \mathrm{C}\right)$ en recipientes de plástico con cierre hermético, etiquetados con la fecha de recolección y almacenamiento, así como el lugar de origen. El tiempo transcurrido del beneficio de la semilla y la prueba de germinación fue de cuatro años.

Parámetros germinativos evaluados. Mediante la prueba de flotabilidad en agua se seleccionaron 400 semillas viables por procedencia, para esto las semillas se colocaron en agua simple y las que flotaron fueron eliminadas. En seguida, cada semilla se sometió a un proceso físico de escarificación, para lo cual se lijó la testa de las semillas hasta que se observó el cotiledón de la semilla (ViverosViveros et al. 2015). Una vez concluida la escarificación, cuatro réplicas de 100 semillas de cada una de las 10 procedencias se pusieron a germinar a temperatura ambiente (varió de 28 a $30^{\circ} \mathrm{C}$ ) en charolas de plástico (con un tamaño de $13 \times 12 \times 7 \mathrm{~cm}$ ), enrolladas con papel absorbente humedecido con agua purificada, en laboratorio bajo un diseño experimental completamente aleatorio. La prueba 
Cuadro 1. Localización geográfica de 10 procedencias de Enterolobium cyclocarpum en la región Costa de Oaxaca, México.

Geographical localization of the 10 Enterolobium cyclocarpum provenances in the Oaxaca Coast Region, Mexico.

\begin{tabular}{|c|c|c|c|c|c|}
\hline Procedencia & Municipio & $\begin{array}{c}\text { Longitud } \\
\mathrm{O}\end{array}$ & $\begin{array}{l}\text { Latitud } \\
\qquad \mathrm{N}\end{array}$ & $\begin{array}{l}\text { Altitud } \\
\mathrm{m}\end{array}$ & Condición \\
\hline Pinotepa de Don Luis & Pinotepa de Don Luis & $97^{\circ} 58^{\prime}$ & $16^{\circ} 26^{\prime}$ & 420 & Potrero \\
\hline Tataltepec & Tataltepec de Valdés & $97^{\circ} 32^{\prime}$ & $16^{\circ} 17^{\prime}$ & 370 & Potrero \\
\hline San Pedro & San Pedro Mixtepec & $97^{\circ} 04^{\prime}$ & $15^{\circ} 58^{\prime}$ & 240 & ZU \\
\hline Pochutla & San Pedro Pochutla & $96^{\circ} 27^{\prime}$ & $15^{\circ} 49^{\prime}$ & 234 & SBC \\
\hline San Francisco & Villa de Tututepec Melchor Ocampo & $97^{\circ} 34^{\prime}$ & $16^{\circ} 08^{\prime}$ & 67 & $\mathrm{ZC}$ \\
\hline Cortijo & Santa María de Cortijo & $98^{\circ} 16^{\prime}$ & $16^{\circ} 27^{\prime}$ & 59 & Potrero \\
\hline Colotepec & Santa María de Colotepec & $96^{\circ} 58^{\prime}$ & $15^{\circ} 53^{\prime}$ & 37 & $\mathrm{ZC}$ \\
\hline Los Limones & Villa de Tututepec Melchor Ocampo & $97^{\circ} 29^{\prime}$ & $16^{\circ} 01^{\prime}$ & 23 & Potrero \\
\hline La Tuza & Santiago de Jalmitepec & $97^{\circ} 51^{\prime}$ & $16^{\circ} 02^{\prime}$ & 15 & SMC \\
\hline El Zarzal & Santiago de Jalmitepec & $97^{\circ} 47^{\prime}$ & $16^{\circ} 04^{\prime}$ & 14 & Potrero \\
\hline
\end{tabular}

ZU: Zona Urbana, SBC: Selva baja caducifolia, ZC: Zona de cultivo, SMC: Selva mediana caducifolia.

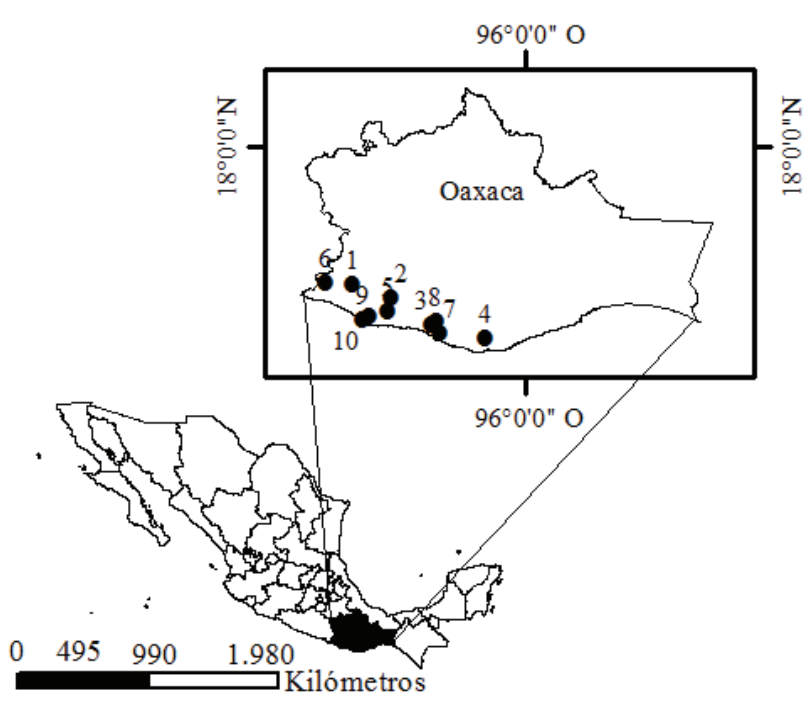

Figura 1. Localización geográfica de 10 procedencias de Enterolobium cyclocarpum en la región Costa de Oaxaca, México.

Geographical localization of the 10 Enterolobium cyclocarpum provenances in the Oaxaca Coast Region, Mexico.

de germinación se mantuvo en observación durante cinco días y el conteo fue diario.

Los parámetros germinativos que se evaluaron fueron la capacidad germinativa, la germinación media diaria, el valor germinativo y el valor pico. Se consideró una semilla germinada cuando la radícula alcanzó un tamaño de al menos $2 \mathrm{~mm}$ (Trindade-Lessa et al. 2015). La capacidad ger- minativa fue el porcentaje de semilla que germinó; la germinación media diaria se obtuvo al dividir los porcentajes de germinación acumulados diariamente entre la longitud en días del periodo de cada evaluación (Czabator 1962); el valor pico fue la germinación máxima acumulada y el valor germinativo se estimó mediante dos métodos, uno mediante la fórmula propuesta por Czabator (1962):

Valor germinativo $=$ Germinación media diaria $\mathrm{x}$ valor pico $[1]$

y el otro, mediante la fórmula propuesta por Djavanshir y Pourbeik (1976):

$$
\text { Valor germinativo }=\left(\frac{\sum D G S}{N}\right) x(G P) x(10)
$$

Donde: DGS $=$ Velocidad de germinación diaria, $\mathrm{N}=$ frecuencia del número de DGS que se calcularon durante la prueba, $\mathrm{GP}=$ porcentaje de germinación al final de la prueba y 10 es una constante.

Variables climáticas. Con la ubicación geográfica y altitud sobre el nivel del mar de cada procedencia se obtuvieron del Laboratorio de Ciencias Forestales de Moscow (Crookston 2006), algunas variables climáticas con importancia ecológica y geográfica para las plantas (Tuhkanen 1980; cuadro 2). De estas variables se derivaron las siguientes, de acuerdo a Sáenz-Romero et al. (2006, 2010) (cuadro 2):

$$
\text { Índice anual de aridez (IAA1) }=\frac{G D 5}{P M A}
$$


Cuadro 2. Variables climáticas de 10 procedencias de E. cyclocarpum de la región Costa de Oaxaca, México.

Climatic variables of the 10 E. cyclocarpum provenances in the Oaxaca Coast Region, Mexico.

\begin{tabular}{lccccccccccc}
\hline Procedencia & TMA & TMAX & TMIN & PMA & PTC & PPV & GD5 & GDPH5 & IAA1 & IAA2 & IAV \\
\hline Pinotepa D.L. & 27,7 & 29,4 & 25,8 & 1.446 & 1.217 & 0,841 & 8.154 & 8.113 & 5,639 & 0,062 & 0,995 \\
Tataltepec & 28,1 & 29,9 & 25,8 & 1.140 & 982 & 0,861 & 8.298 & 8.298 & 7,279 & 0,079 & 1,000 \\
San Pedro & 27,5 & 28,8 & 25,2 & 985 & 834 & 0,846 & 8.093 & 8.053 & 8,216 & 0,091 & 0,995 \\
Pochutla & 27,0 & 28,0 & 24,5 & 1.168 & 992 & 0,849 & 7.921 & 7.843 & 6,762 & 0,076 & 0,990 \\
San Francisco & 28,5 & 30,0 & 26,1 & 1.083 & 925 & 0,854 & 8.468 & 8.468 & 7,819 & 0,084 & 1,000 \\
Cortijo & 28,5 & 29,9 & 25,8 & 1.031 & 850 & 0,824 & 8.450 & 8.470 & 8,196 & 0,089 & 1,002 \\
Colotepec & 27,9 & 29,3 & 25,4 & 840 & 726 & 0,864 & 8.232 & 8.232 & 9,800 & 0,108 & 1,000 \\
Limones & 28,5 & 29,9 & 26,1 & 1.070 & 909 & 0,849 & 8.449 & 8.427 & 7,896 & 0,085 & 0,997 \\
La Tuza & 28,4 & 29,5 & 26,2 & 1.064 & 902 & 0,847 & 8.428 & 8.428 & 7,921 & 0,086 & 1,000 \\
El Zarzal & 28,4 & 29,7 & 26,2 & 1.069 & 908 & 0,849 & 8.440 & 8.440 & 7,895 & 0,085 & 1,000 \\
\hline
\end{tabular}

D.L.: de Don Luis, TMAX: temperatura máxima, TMIN: temperatura mínima, TMA: temperatura media anual, PMA: precipitación media anual, PTC: precipitación total en la estación de crecimiento, GD5: grados días $>5^{\circ} \mathrm{C}$, GDPH5: grados días $>5^{\circ} \mathrm{C}$ en el periodo libre de heladas, IAA1: índice anual de aridez fórmula [3], IAA2: índice anual de aridez fórmula [4], IAV: índice de aridez en verano fórmula [5], PPV: proporción de la precipitación total que cae durante el verano fórmula [6].

$$
\begin{aligned}
\text { Índice anual de aridez (IAA2) } & =\frac{\sqrt{G D 5}}{P M A} \\
\text { Índice de aridez en verano (IAV) } & =\frac{G D P H 5}{P T C}
\end{aligned}
$$

Proporción de la precipitación total que cae durante el verano (PPV) $=\frac{P T C}{P M A}$

Donde: PMA: Precipitación media anual, PTC: Precipitación total en la estación de crecimiento GD5: Grados días $>5{ }^{\circ} \mathrm{C}$, GDPH5: Grados días $>5^{\circ} \mathrm{C}$ en el periodo libre de heladas.

Análisis estadísticos. Se realizaron análisis de varianza para las variables evaluadas con el modelo:

$$
Y_{i j}=\mu+P_{i}+R_{j}+E_{i j}
$$

Donde: $Y_{i j}$ es el valor de la ij-ésima observación, $\mu$ es la media general, $P_{i}$ es el efecto de la i-ésima procedencia, $R_{j}$ es el efecto de j-ésima repetición y $E_{i j}$ es el error experimental.

La transformación con el arcoseno de la raíz de los datos originales divididos entre 100 se realizó para la capacidad germinativa debido a que los datos originales no cumplieron con los supuestos de normalidad estadística. Esta transformación dio un mayor ajuste en la prueba de Shapiro-Wilk. Las demás variables si cumplieron con los supuestos de normalidad, valor de $P>0,05$ en la prueba de Shapiro-Wilk. Los análisis se realizaron mediante el procedimiento GLM de SAS (SAS Institute 2004). Los componentes de varianza asociados a cada fuente de variación se estimaron mediante el procedimiento VARCOMP, opción REML (SAS Institute 2004). La prueba de comparación de medias de Tukey se realizó cuando existieron diferencias significativas entre procedencias.

Para conocer las mejores procedencias considerando los parámetros germinativos en conjunto, se llevó a cabo un análisis multivariado de componente principales (ACP). El componente principal uno se obtuvo como nueva variable resultante del ACP. Después las diferencias entre procedencias del componente principal uno (CP1) se determinaron con pruebas no paramétricas de varianza y comparaciones múltiples de rangos de Kruskal-Wallis usando en programa estadístico InfoStat (Di Rienzo et al. 2015).

El agrupamiento de las procedencias según los valores de los parámetros germinativos se realizó con la agrupación jerárquica de Ward y la distancia euclidiana. Para conocer la asociación de los parámetros germinativos con las variables geográficas y climáticas de cada procedencia se realizó un análisis de correlación simple con el procedimiento CORR de SAS (SAS Institute 2004).

\section{RESULTADOS}

Variación geográfica de la germinación. La germinación de semilla de E. cyclocarpum inició a partir del primer día de establecido el ensayo (figura 2). Independientemente de la procedencia, la capacidad máxima de germinación ocurrió al tercer día. Después no ocurrieron incrementos apreciables en la germinación, es decir, el porcentaje máximo de germinación se alcanzó rápidamente y luego se estabilizó. 
Por otra parte, existieron diferencias significativas entre procedencias en todos los parámetros de germinación $(P<0,01)$ (cuadro 3). En promedio la aportación de las procedencias a la varianza total fue cercana al $81 \%$ mientras que la aportación del error fue del $19 \%$. La mayor contribución de las procedencias a la varianza total se dio en la capacidad germinativa y el valor pico, con valores cercanos al 94 y $91 \%$, respectivamente; mientras que la menor contribución de las procedencias a la varianza se dio en el valor germinativo [tipo 1] (50 \%). La aportación de las procedencias y del error a la varianza total fue igual solo para para el vigor germinativo [tipo 1], mientras que en los demás parámetros germinativos la aportación de las procedencias fue mayor a la del error (cuadro 3).

Las procedencias de Pinotepa de Don Luis, Tataltepec y Colotepec presentaron los mayores porcentajes de germinación, de aproximadamente 90 a $80 \%$, mientras que la procedencia de Cortijo fue la de menor capacidad germinativa (cuadro 4).

En lo referente al valor pico, las procedencias de Pinotepa de Don Luis y la de Colotepec presentaron los valores pico más elevados, 28,5 y 24,8 , respectivamente; mientras que la procedencia Cortijo mostró el valor pico menor (aproximadamente 9, cuadro 4).

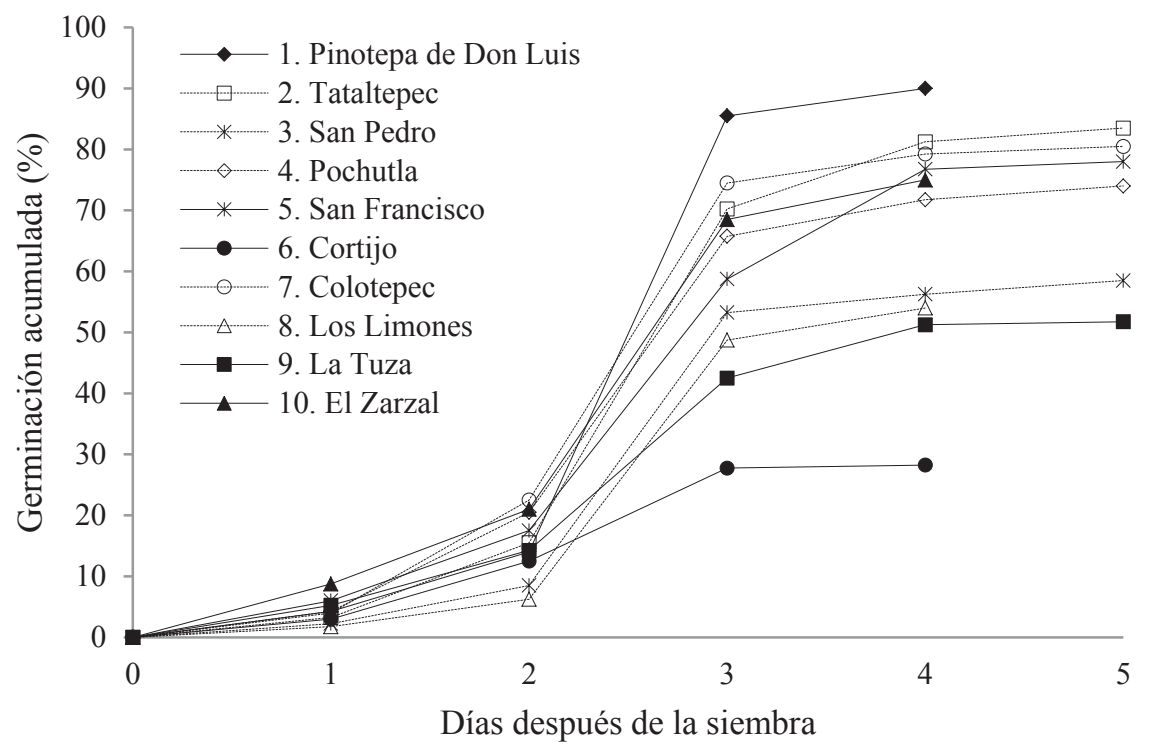

Figura 2. Curvas de germinación acumulada de semillas de Enterolobium cyclocarpum de 10 procedencias de la región Costa de Oaxaca, México.

Accumulated germination curves of Enterolobium cyclocarpum seeds of the 10 Enterolobium cyclocarpum provenances in the Oaxaca Coast Region, Mexico.

Cuadro 3. Nivel de significancia $(P)$ y componentes de varianza (\%) de los parámetros de la germinación en semilla de $E$. cyclocarpum de la región Costa de Oaxaca, México.

Significance level $(P)$ and percentage of contribution to total variance (\%) of germination parameters of Enterolobium cyclocarpum seeds from the Oaxaca Coast Region, Mexico.

\begin{tabular}{ccccc}
\hline \multirow{2}{*}{ Variables } & \multicolumn{3}{c}{ Valor $P$} & \multicolumn{2}{c}{ Componentes de varianza (\%) } \\
\cline { 2 - 5 } & Procedencia & Repetición & Procedencia & Error \\
\hline Capacidad germinativa & $<0,0001$ & 0,8780 & 94,34 & 5,66 \\
Valor pico & $<0,0001$ & 0,7269 & 91,25 & 8,75 \\
Germinación media diaria & $<0,0001$ & 0,9895 & 81,97 & 18,03 \\
Valor germinativo [1] & $<0,0001$ & 0,9488 & 50,00 & 50,00 \\
Valor germinativo [2] & $<0,0001$ & 0,9454 & 86,31 & 13,69 \\
Promedio & - & - & 80,77 & 19,23 \\
\hline
\end{tabular}


Cuadro 4. Valores promedios de los parámetros germinativos, agrupamiento Tukey $(P \leq 0,05)$ y comparaciones múltiples de KruskalWallis $(P \leq 0,05)$ de 10 procedencias de Enterolobium cyclocarpum de la región Costa de Oaxaca, México.

Means of germination parameters, Tukey test $(P \leq 0.05)$ and Kruskal-Wallis multiple-comparisons test $(P \leq 0.05)$ of the 10 Enterolobium cyclocarpum provenances in the Oaxaca Coast Region, Mexico.

\begin{tabular}{|c|c|c|c|c|c|c|}
\hline \multirow{2}{*}{ Procedencias } & \multicolumn{5}{|c|}{ Parámetros germinativos } & \multirow{2}{*}{$\mathrm{CP} 1$} \\
\hline & CG & VP & GMD & VG [1] & VG [2] & \\
\hline Pinotepa de Don Luis & $90,0 \mathrm{a}$ & $28,5 \mathrm{a}$ & $22,5 \mathrm{a}$ & $642,1 \mathrm{a}$ & $35,0 \mathrm{a}$ & $3,54 \mathrm{f}$ \\
\hline Tataltepec & $81,7 \mathrm{ab}$ & $23,4 b c$ & $18,3 \mathrm{ab}$ & $428,8 b$ & $27,9 \mathrm{ab}$ & $1,54 \mathrm{ef}$ \\
\hline Colotepec & $80,5 \mathrm{ab}$ & $24,8 \mathrm{ab}$ & $16,1 \mathrm{cde}$ & $401,3 b$ & $27,6 a b$ & $1,27 \mathrm{cdef}$ \\
\hline San Francisco & $78,0 \mathrm{~b}$ & 20,0 cde & $17,6 \mathrm{bc}$ & $356,9 \mathrm{bc}$ & $22,6 \mathrm{bcd}$ & $0,64 \mathrm{abcde}$ \\
\hline El Zarzal & $75,0 \mathrm{~b}$ & $22,8 b c$ & $18,7 \mathrm{ab}$ & $429,0 \mathrm{~b}$ & $26,7 \mathrm{ab}$ & 1,32def \\
\hline Pochutla & $72,0 \mathrm{~b}$ & $21,9 \mathrm{bcd}$ & $17,0 \mathrm{bcd}$ & $374,2 b$ & $23,4 \mathrm{bc}$ & $0,67 \mathrm{bcdef}$ \\
\hline San Pedro & $56,7 \mathrm{c}$ & $17,7 \mathrm{def}$ & $12,7 \mathrm{def}$ & $228,1 \mathrm{~cd}$ & 14,3 cde & $-1,36 \mathrm{abcd}$ \\
\hline Limones & $54,0 \mathrm{c}$ & $16,2 \mathrm{ef}$ & $13,5 \mathrm{cde}$ & 221,0 cde & 13,3 def & $-1,52 \mathrm{abc}$ \\
\hline La Tuza & $51,7 \mathrm{c}$ & $14,1 f$ & $12,3 \mathrm{ef}$ & $179,8 \mathrm{de}$ & $11,4 \mathrm{ef}$ & $-2,08 \mathrm{ab}$ \\
\hline Cortijo & $28,2 \mathrm{~d}$ & $9,2 \mathrm{~g}$ & $8,8 \mathrm{f}$ & $82,5 \mathrm{e}$ & $4,6 f$ & $-4,02 \mathrm{a}$ \\
\hline
\end{tabular}

Las procedencias se agruparon de mayor a menor capacidad germinativa, $\mathrm{CG}=$ capacidad germinativa, $\mathrm{VP}=\mathrm{valor}$ pico, $\mathrm{GMD}=$ germinación media diaria, $\mathrm{VG}[1]$ = vigor germinativo [1], VG[2] = vigor germinativo [2], CP1 = componente principal 1 . Letras diferentes en cada columna indican diferencias con $P \leq 0,05$.

Las procedencias de Pinotepa de Don Luis, El Zarzal y Tataltepec, presentaron la mayor germinación media diaria, del 18,3 al 22,5\%, aproximadamente; la procedencia del Cortijo se diferenció de todas las demás procedencias ya que presentó la menor germinación media diaria (cuadro 4).

Referente al valor germinativo [tipo 1], la procedencia de Pinotepa de Don Luis fue la de mayor valor germinativo; mientras que los Limones, la Tuza y el Cortijo fueron las de menores valores (cuadro 4). Por otra parte, en cuanto al valor germinativo [tipo 2], las procedencias de Pinotepa de Don Luis, Tataltepec y Colotepec presentaron los mayores valores (de 35 a 27); y las de los Limones, la Tuza y el Cortijo presentaron los valores menores. Las dos estimaciones del valor germinativo [tipos 1 y 2] indicaron que la procedencia de Pinotepa de Don Luis fue la que alcanzó los mejores resultados, sólo que el tipo 2 pareciera ser más flexible ya que además de la procedencia de Pinotepa de Don Luis incluye a otras tres procedencias.

En el análisis de componentes principales, el componente principal uno (CP1) de los parámetros germinativos explicó el $97 \%$ de la varianza; todos los parámetros germinativos contribuyeron con valores similares (entre 0,44 y 0,45 ) para explicar la variabilidad. El CP1 de los parámetros germinativos presentó diferencias estadísticas $(P<0,0001)$ entre las procedencias, con valores de $-4,02$ (Cortijo) a 3,54 (Pinotepa de Don Luis). Las procedencias con valores positivos o negativos del CP1 (cuadro 4) correspondieron a cada uno de los grupos obtenidos por el análisis de agrupamiento, uno formado por las proce- dencias con valores positivos y el otro con las de valores negativos (figura 3 ).

Las comparaciones múltiples de rangos de KruskalWalllis del CP1 sugiere que las procedencias de Pinotepa de Don Luis, Tataltepec, el Zarzal, Colotepec y Pochutla se diferenciaron en cuanto a los parámetros germinativos

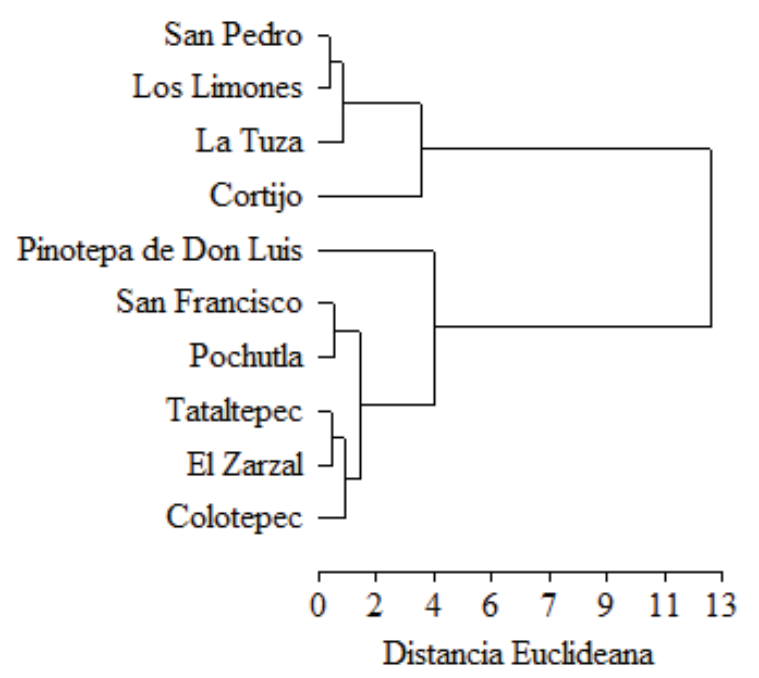

Figura 3. Agrupamiento de 10 procedencias de Enterolobium cyclocarpum de la región Costa de Oaxaca, México, utilizando la distancia euclidiana.

Cluster for 10 Enterolobium cyclocarpum provenances in the Oaxaca Coast Region, Mexico, using the Euclidean distance. 
evaluados de manera conjunta, de las de San Francisco, San Pedro, los Limones, la Tuza y el Cortijo (cuadro 4).

El análisis multivariado indicó que las procedencias se agruparon en dos grandes grupos, el primero formado por las procedencias con mejores resultados en cuanto a los parámetros germinativos evaluados (Pinotepa de Don Luis, San Francisco, Pochutla, Tataltepec, El Zarzal y Colotepec) y, el segundo grupo integrado por las procedencias con los resultados más bajos (San Pedro, Los Limones, La Tuza y El Cortijo). Dentro de cada uno de estos dos grupos se destacó una procedencia, la de Pinotepa de Don Luis dentro del primer grupo por presentar los mejores resultados, y del segundo grupo la del Cortijo por presentar los resultados más bajos, lo que concuerda con los análisis univariados (figura 3).

Asociación entre los parámetros germinativos y las variables geográficas. No se encontró asociación entre los parámetros germinativos y las variables geográficas (altitud, latitud y longitud) de las diez procedencias evaluadas de E. cyclocarpum $(P \leq 0,05)$.

Asociación entre los parámetros germinativos y las variables climáticas. La asociación entre la capacidad germinativa de las semillas de E. cyclocarpum y la proporción de precipitación que ocurre durante el verano en cada una de las procedencias de recolecta de la especie fue positiva y significativa ( $\mathrm{r}=0,71, P=0,0216$; cuadro 5, figura 4); es decir, entre mayor fue la proporción (respecto a la precipitación total) de lluvia que cae durante la estación de crecimiento, mayor fue la capacidad germinativa. De la misma manera, existió asociación positiva y significativa entre la germinación media diaria y la precipitación que cae durante los meses de abril a septiembre, en la estación de crecimiento ( $r=0,66, P=0,0382$, cuadro 5, figura 4).
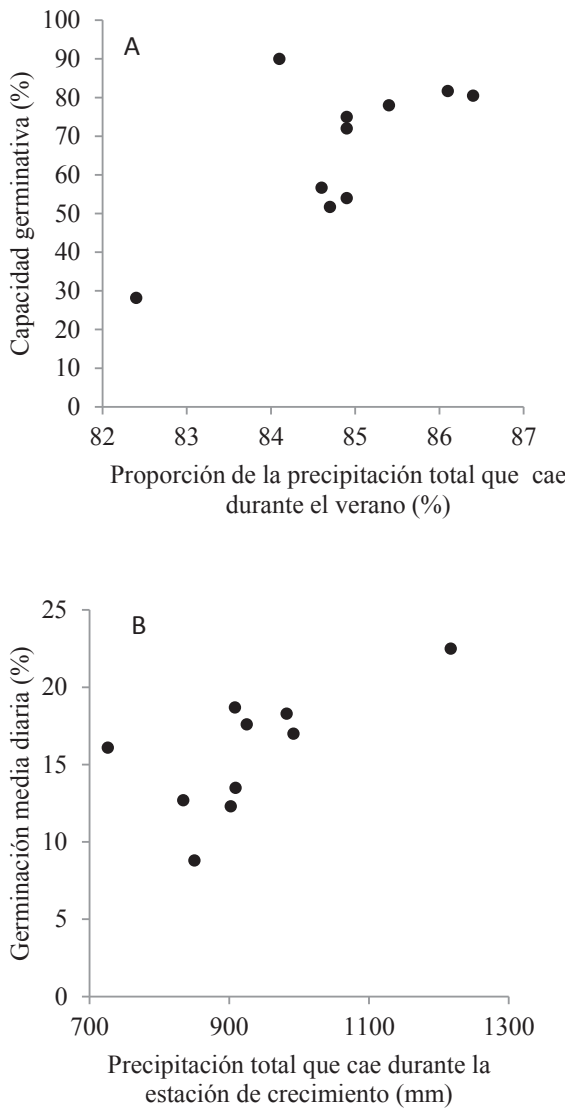

Figura 4. Relación entre A) la capacidad germinativa y la proporción de precipitación que cae durante el verano y B) la germinación media diaria y la precipitación que cae durante la estación de crecimiento en 10 procedencias de Enterolobium cyclocarpum de la región costa de Oaxaca, México.

Relationship between A) the germinative capacity and proportion of precipitation that falls during the summer and B) the mean daily germination and total precipitation in the growing season.

Cuadro 5. Coeficiente de correlación de Pearson (r) de la asociación entre los parámetros germinativos y las variables climáticas en 10 procedencias de Enterolobium cyclocarpum de la región costa de Oaxaca, México.

Pearson's correlation coefficient ( $\mathrm{r}$ ) of the relationship between the germination parameters and climatic variables of the 10 Enterolobium cyclocarpum provenances in the Oaxaca Coast Region, Mexico.

\begin{tabular}{lccccccccccc}
\hline \multirow{2}{*}{ Variable } & \multicolumn{10}{c}{ Variable climática } \\
\cline { 2 - 11 } & TMA & TMAX & TMIN & PMA & PTC & GD5 & GDPH5 & IAA[3] & IAA[4] & IAV & PPV \\
\hline CG & $-0,43 \mathrm{~ns}$ & $-0,32 \mathrm{~ns}$ & $-0,11 \mathrm{~ns}$ & $0,45 \mathrm{~ns}$ & $0,42 \mathrm{~ns}$ & $-0,34 \mathrm{~ns}$ & $-0,34 \mathrm{~ns}$ & $-0,32 \mathrm{~ns}$ & $-0,30 \mathrm{~ns}$ & $-0,26 \mathrm{~ns}$ & $0,71^{*}$ \\
VP & $-0,50 \mathrm{~ns}$ & $-0,43 \mathrm{~ns}$ & $-0,20 \mathrm{~ns}$ & $0,41 \mathrm{~ns}$ & $0,46 \mathrm{~ns}$ & $-0,45 \mathrm{~ns}$ & $-0,45 \mathrm{~ns}$ & $-0,34 \mathrm{~ns}$ & $-0,31 \mathrm{~ns}$ & $-0,34 \mathrm{~ns}$ & $0,61 \mathrm{~ns}$ \\
GMD & $-0,39 \mathrm{~ns}$ & $-0,26 \mathrm{~ns}$ & $0,09 \mathrm{~ns}$ & $0,61 \mathrm{~ns}$ & $0,66^{*}$ & $-0,29 \mathrm{~ns}$ & $-0,30 \mathrm{~ns}$ & $-0,55 \mathrm{~ns}$ & $-0,54 \mathrm{~ns}$ & $-0,30 \mathrm{~ns}$ & $0,48 \mathrm{~ns}$ \\
VG[1] & $-0,32 \mathrm{~ns}$ & $-0,47 \mathrm{~ns}$ & $-0,14 \mathrm{~ns}$ & $0,57 \mathrm{~ns}$ & $0,61 \mathrm{~ns}$ & $-0,51 \mathrm{~ns}$ & $-0,51 \mathrm{~ns}$ & $-0,50 \mathrm{~ns}$ & $-0,46 \mathrm{~ns}$ & $0,40 \mathrm{~ns}$ & $0,39 \mathrm{~ns}$ \\
VG[2] & $-0,46 \mathrm{~ns}$ & $-0,34 \mathrm{~ns}$ & $-0,08 \mathrm{~ns}$ & $0,45 \mathrm{~ns}$ & $0,50 \mathrm{~ns}$ & $-0,37 \mathrm{~ns}$ & $-0,36 \mathrm{~ns}$ & $-0,38 \mathrm{~ns}$ & $-0,36 \mathrm{~ns}$ & $-0,25 \mathrm{~ns}$ & $0,59 \mathrm{~ns}$ \\
\hline
\end{tabular}

$\mathrm{CG}=$ capacidad germinativa, $\mathrm{VP}=$ valor pico, $\mathrm{GMD}$ = germinación media diaria, $\mathrm{VG}[1]$ = vigor germinativo [1], $\mathrm{VG}[2]$ = vigor germinativo [2], TMAX: temperatura máxima, TMIN: temperatura mínima, TMA: temperatura media anual, PMA: precipitación media anual, PTC: precipitación total en la estación de crecimiento GD5: grados días $>5^{\circ} \mathrm{C}$, GDPH5: grados días $>5^{\circ} \mathrm{C}$ en el periodo libre de heladas, IAA[3]: índice anual de aridez [3], IAA[4]: índice anual de aridez [4], IAV: índice de aridez en verano [5], PPV: proporción de la precipitación total que cae durante el verano [6], $*=P<0,05$, ns $=$ no significativo. 


\section{DISCUSIÓN}

Variación geográfica de la germinación. Bertsch (1985) encontró un patrón similar en las curvas de germinación de semilla de esta misma especie, pero de procedencias de Costa Rica y germinadas en vivero, aunque las semillas germinaron a partir de la segunda semana de sembradas, e independientemente de las procedencias la capacidad máxima de germinación ocurrió en la tercera semana. La diferencia en el tiempo de germinación entre lo determinado en el presente trabajo y lo reportado por Bertsch (1985) puede ser que la germinación en este trabajo se evaluó en el laboratorio, con condiciones más contraladas que en el vivero. También puede ser a que el experimento de Bertsch (1985) se realizó sembrando las semillas en sustrato, por lo que no se pudo observar cuándo realmente se inició la germinación, solo hasta que las plántulas fueron visibles al emerger del sustrato, aunque se desconoce la profundidad a la que fue sembrada la semilla.

No es sorprendente la variación encontrada de los parámetros germinativos a nivel de procedencias debido a la amplia distribución de la especie a lo largo de la costa de Oaxaca, México, la que debe estar relacionada con la variación ambiental en las procedencias evaluadas. Diversos trabajos en plantas, reportan variación entre procedencias en parámetros germinativos (Bertsch 1985, Davidson et al. 1996, Benowicz et al. 2000, 2001, Juárez-Agis et al. 2006, Mápula-Larreta et al. 2008). La variación encontrada en el presente trabajo puede ser de origen genético, sin embargo, también se puede deber a factores ambientales, causados por condiciones locales en donde se desarrolló y maduró la semilla (Mamo et al. 2006). La baja germinación que mostró la procedencia del cortijo puede tener repercusiones en la regeneración, ocasionando que esta sea escasa, lo que en consecuencia puede provocar problemas en la permanencia y conservación de la procedencia en un futuro.

Al igual que en este estudio, Davidson et al. (1996), Bertsch (1985), Juárez-Agis et al. (2006) y Mamo et al. (2006) encontraron diferencias significativas entre procedencias para la capacidad germinativa en E. cyclocarpum, Abies amabilis, Pseudostuga menziesii y Juniperus procera Endl., respectivamente; mientras que Mápula-Larreta et al. (2008) reportaron diferencias significativas entre procedencias para el vigor germinativo en $P$. menziesii y Benowicz et al. $(2000,2001)$ en la capacidad germinativa, valor pico y valor germinativo de Alnus sinuata y Betula papyrifera.

Asociación entre los parámetros germinativos y las variables geográficas. La falta de asociación entre los parámetros germinativos y las variables geográficas indica que la altitud, latitud y longitud de origen de las semillas de E. cyclocarpum de la Costa de Oaxaca, México no tienen efecto y no definen algún gradiente sobre los parámetros germinativos evaluados. Lo anterior coincide con lo reportado en A. amabilis, P. menziesii y J. procera, en los que no se encontró asociación significativa entre la capacidad germinativa y las variables geográficas (Davidson et al. 1996, Juárez-Agis et al. 2006, Mamo et al. 2006).

Asociación entre los parámetros germinativos y las variables climáticas. En cuanto a la asociación positiva significativa entre la capacidad germinativa de las semillas de E. cyclocarpum y la proporción de precipitación que cae durante el verano, y entre la germinación media diaria y la precipitación total que cae en la estación de crecimiento, Bertsch (1985) menciona que posiblemente la capacidad germinativa de semilla de E. cyclocarpum sea mayor en lugares o zonas donde la precipitación es mayor. E. cyclocarpum es una especie estacional y de manera natural habita lugares caracterizados por alternar periodos de sequía con el de lluvias, por lo que la semilla de esta especie debe aprovechar el periodo del año en que cae mayor proporción de precipitación para completar la germinación.

En especies de clima templado frío se ha encontrado que los parámetros de germinación están fuertemente asociados a los regímenes de temperatura de los sitios de recolección de semillas (Benowicz et al. 2001). Sin embargo, en el caso de E. cyclocarpum, una especie tropical que crece sin presencia de heladas, deja de tener relevancia mayor la temperatura, y toma importancia la precipitación.

Otro factor que puede estar influyendo en la diferenciación de las procedencias y que no fue evaluado en el presente trabajo es el efecto materno de las semillas. Existen evidencias que indican que efectos maternos, como la posición de la semilla en el fruto y del fruto en el árbol, la edad del árbol madre, y el tamaño de la semilla influyen en la germinación de la semilla (Gutterman 2000). Como las semillas fueron recolectadas en bosques naturales de la especie, es de esperar que los árboles madres no fueran de la misma edad.

\section{CONCLUSIONES}

La germinación de Enterolobium cyclocarpum evaluada por los parámetros germinativos difiere entre procedencias (entre 28 y $90 \%$ ). En las procedencias que presentan bajo porcentaje de germinación, posiblemente en un futuro pueden presentar problemas en su repoblación. La procedencia que muestra los mayores valores de los parámetros germinativos evaluados fue la de Pinotepa de Don Luis. Las variables geográficas del origen de las semillas no tienen efecto sobre los parámetros germinativos. En cambio, la precipitación que cae durante el periodo de crecimiento influye en la capacidad germinativa y germinación media diaria; esta asociación muestra que, a mayor precipitación, mayor capacidad germinativa y mayor geminación media diaria de semillas de E. cyclocarpum en la costa de Oaxaca, México. 


\section{AGRADECIMIENTOS}

Los autores agradecen a René Robles Silva y María Luisa Hernández Hernández por la donación y recolecta de semillas.

\section{REFERENCIAS}

Benítez-Badillo G, MT Pulido-Salas, M Equihua-Zamora. 2004. Árboles multiusos nativos de Veracruz para reforestación: restauración y plantaciones. Xalapa, Veracruz, México. Instituto de Ecología, A.C. SIGOLFO, Comisión Nacional Forestal. 288 p.

Benowicz A, YA El-Kassaby, RD Guy, CC Ying. 2000. Sitka Alder (Alnus sinuate Rydb): genetic diversity in germination, frost hardiness and growth attributes. Silvae Genetica 49(45): 206-212.

Benowicz A, R Guy, MR Carlson, YA El-Kassaby. 2001. Genetic variation among paper birch (Betula papyrifera Marsh.) populations in germination, frost hardiness, gas exchange and growth. Silvae Genetica 50(1): 7-13.

Bertsch CS. 1985. Germinación y crecimiento del guanascaste Enterolobium cyclocarpum (Jacq.) Griseb en vivero y en el campo. Tesis Licenciada en Biología. San José, Costa Rica. Facultad de Ciencias, Escuela de Biología, Universidad de Costa Rica. 68 p.

Buch-Texaj MS. 1999. Evaluación de la germinación de semillas pretratadas de Caesalpinia velutina (Britt. et Rose) Standl (Aripin), Leucaena leucocephala (Lam.) de Wit. (Leucaena) y Enterolobium cyclocarpum (Jacq.) Griseb. (conacaste) almacenadas a dos temperaturas. Tesis Ingeniero Agrónomo. Guatemala, Guatemala. Facultad de Agronomía, Instituto de Investigaciones Agronómicas, Universidad San Carlos de Guatemala. 70 p.

Cano-Hernández A, S Romero-Guillot. 2012. Uso alimentario y prácticas culinarias del guanacastle (Enterolobium cyclocarpum) en el municipio de Santa María Huatulco, Oaxaca. Ciencia y Mar 16(48): 37-43.

CATIE (Centro Agronómico Tropical de Investigación y Enseñanza, CR). 1997. Biología de semillas forestales. Turrialba, Costa Rica. CATIE. 32 p. (Material de Enseñanza No. 36).

Crookston N. 2006. Research on forest climate change: Potential effects of global warming on forests and plant climate relationships in western North America and Mexico. Consultado 15 de ene. 2013. Disponible en http://forest.moscowfsl. wsu.edu/climate/customData/

Czabator FJ. 1962. Germination value: An index combining speed and completeness of pine seed germination. Forest Science 8(4): 386-396.

Davidson RH, DGW Edwards, O Sziklai, YA El-Kassaby. 1996. Genetic variation in germination parameters among populations of pacific silver fir. Silva Genetica 45(2-3): 165-171.

Di Rienzo JA, F Casanoves, MG Balzarini, L Gonzalez, M Tablada, CW Robledo. 2015. InfoStat versión 2015. Consultado 21 de dic. 2015. Disponible en http://www.infostat.com.ar

Djavanshir K, H Pourbeik. 1976. Germination value - a new formula. Silvae Genetica 25(2): 79-83.

Espejel I, E Martínez. 1979. El guanacaste. Jalapa, Veracruz, México. Instituto Nacional de Investigaciones sobre Recursos Botánicos. 4 p. (Comunicado 33).
Flores-Flores C, J López-Upton, S Valencia-Manzo. 2014. Manual técnico para el establecimiento de ensayos de procedencias y progenies. Zapopan, Jalisco, México. Comisión Nacional Forestal. 152 p.

Ginwal HS, SS Phartyal, PS Rawat, RL Srivastava. 2005. Seed sources variation in morphology, germination and seedling growth of Jatropha curcas Linn. in Central India. Silvae Genetica 54(2): 76-80.

Gutterman Y. 2000. Maternal effects on seeds during development. In Fenner M ed. Seeds: The ecology of regeneration in plant communities. Wallingford, UK. CAB International. p. 27-59.

Juárez-Agis A, J López-Upton, JJ Vargas-Hernández, C SáenzRomero. 2006. Variación geográfica en la germinación y crecimiento inicial de plántulas de Pseudotsuga menziesii de México. Agrociencia 40(6): 783-792.

Mamo N, M Mihretu, M Fekadu, M Tigabu, D Teketay. 2006. Variation in seed and germination characteristic among $\mathrm{Ju}$ niperus procera populations in Ethiopia. Forest Ecology and Management 225: 320-327.

Manzanilla BH, M Martínez D, A Moreno M. 2001. Monografías de especies nativas promisorias para el establecimiento de plantaciones comerciales en Jalisco cedro rojo y rosa morada. Guadalajara, México. Programa de Desarrollo Forestal de Jalisco. 57 p. (Documento Técnico 31).

Mápula-Larreta M, J López-Upton, JJ Vargas-Hernández, A Hernández-Livera. 2008. Germinación y vigor de semillas en Pseudotsuga menziesii de México. Ra Ximhai 4(1): 119134.

Sáenz-Romero C, R Guzmán-Reyna, GE Rehfeldt. 2006. Altitudinal genetic variation among Pinus oocarpa populations in Michoacán, México; implications for seed zoning, conservation of forest genetic resources, tree breeding and global warming. Forest Ecology and Management 229(1-3): 340-350.

Sáenz-Romero C, GE Rehfeldt, NL Crookston, D Pierre, R StAmant, J Beaulieu, B Richardson. 2010. Contemporary and projected spline climate surfaces for Mexico and their use in understanding climate-plant relationships. Climatic Change 102: 595-623.

SAS Institute Inc. 2004. SAS/STAT 9.1 User's Guide. North Carolina, USA. SAS Institute Inc. Cary.

Serratos-Arévalo JC. 2001. Extracción y caracterización de proteínas de la almendra de las semillas maduras de Enterolobium cyclocarpum para su aprovechamiento alimenticio. Tesis Doctor en Ciencias. Colima, México. Posgrado Interinstitucional en Ciencias Pecuarias. Universidad de Colima. $67 \mathrm{p}$.

Trindade-Lessa BF da, JP Nobre-de Almeida, C Lobo-Pinheiro, F Melo-Gomes, S Medeiros-Filhos. 2015. Germinación y crecimiento de plántulas of Entelobium contortisiliquum en función del peso de la semilla y las condiciones de temperatura y luz. Agrociencia 40(3): 315-327.

Tuhkanen S. 1980. Climatic parameters and indices in plant geography. Acta Phytogeographica Suecica 67: 1-105.

Vázquez-Yanez C, AI Batis-Muñoz, MI Alcocer-Silva, M GualDíaz, C Sánchez-Dirzo. 1999. Árboles y arbustos potencialmente valiosos para la restauración ecológica y la reforestación. México DF, México. CONABIO. 13 p. (Reporte técnico del proyecto J084).

Viveros-Viveros H, JD Hernández-Palmeros, MV Velasco-Gar- 
cía, R Robles-Silva, C Ruiz-Montiel, A Aparicio-Rentería, M de J Martínez-Hernández, J Hernández-Villa, ML Hernández Hernández. 2015. Análisis de semillas, tratamientos pregerminativos de Enterolobium cyclocarpum (Jacq.) Gri- seb. y su crecimiento inicial. Revista Mexicana de Ciencias Forestales 30: 52-65.

Zobel B, J Talbert. 1992. Técnicas de mejoramiento genético de árboles forestales. México DF, México. Limusa. 545 p.

Recibido: 09.11.16

Aceptado: 16.03.17 